\title{
Clinical utility of metagenomic next-generation sequencing in suspected central nervous system infectious pediatric patients with empirical treatment: a cohort study
}

\section{Bin-Fang Guo}

Cangzhou Central Hospital

Meng-Xiao Ding

Hebei Medical University

Jing-Ru Xue

Hebei Medical University

Dan-Ni Yan

Hebei Medical University

Su-Zhen Sun ( $\square$ sunsuzhen2004@126.com )

Children's Hospital of Hebei Province

\section{Research Article}

Keywords: Metagenomics next generation sequencing, Central nervous system infection, Diagnosis, Clinical utility

Posted Date: March 11th, 2021

DOl: https://doi.org/10.21203/rs.3.rs-275456/v1

License: (c) (i) This work is licensed under a Creative Commons Attribution 4.0 International License.

Read Full License 


\section{Abstract}

Background: Metagenomic next generation sequencing (mNGS) is becoming an increasingly available diagnostic method used to identify a broad range of pathogens. However, the optimal role of mNGS in clinical diagnostic schema remains uncertain, especially in pediatric patients suspected central nervous system (CNS) infection and treated with empirical antibiotic. The purpose of this study was to investigate the usefulness of cerebrospinal fluid (CSF) mNGS in the pediatric patients.

Methods: We performed a retrospective review of suspected CNS infection patients who had CSF mNGS test from April 2019 to December 2020. Results and clinical impact of mNGS test were collected. We investigated the usefulness of CSF mNGS in clinical impact and diagnosis.

Results: We enrolled 57 pediatric patients with empirical treatment. A total of 39 CNS infection patients were diagnosed, and 27 patients were identified by mNGS that only 2 of 27 were co-identified by CSF culture. In all of the patients, $75.4 \%$ (45 cases) had changed (addition or de-escalation) in antimicrobial therapy according to the results of the mNGS test. By each CSF mNGS test as a whole, the positive and negative percent agreement were $69.2 \%$ (95\% Cl: 54.1-84.4\%) and 61.1\% (95\% Cl: 36.2-86.1\%), respectively, and true negatives of negative mNGS tests were $50 \%$ (95\% Cl: $27.3-72.7 \%$ ).

Conclusion: In this study, CSF mNGS test improved the diagnosis of neurologic infections and adjusted antibiotic therapy in the vast majority of cases. Consequently, for patients with empirical treatment, CSF mNGS should be used more in pathogen diagnosis and clinical therapy.

\section{Background}

Central nervous system (CNS) infections are a cause of morbidity and mortality in children. Estimated incidence of acute encephalitis syndrome in children is 10.5/100000[1]. For these patients, effective treatment is critical based on timely identification of causative pathogens [2]. But the pathogen of meningitis is not identified in approximately $50 \%-60 \%$ of patients [3-5]. The most commonly method for pathogen detection is cerebrospinal fluid (CSF) culture. Traditional CSF culture can only identify approximately $30-40 \%$ of CNS infections [6]. Other methods for pathogen detection (such as tissue biopsy, Xpert MTB/RIF Ultra, loop-mediated isothermal amplification, and Filmarray meningitis/encephalitis Panel) can improve diagnostic ability [6, 7], but they are restricted to a limited spectrum of pathogens. Routine microbiologic testing is often deficient to detect all neuroinvasive pathogens. Recent reports found that metagenomic next generation sequencing (mNGS) can be used to identify pathogens in cerebrospinal fluid samples which were not found by conventional microbiological methods [8-10].

mNGS is a promising microbial detection technology for the diagnosis of infectious disease [11], because a wide-ranging spectrum of potential causes - bacterial, viral, fungal, and parasitic - can be independent identified by a single assay $[12,13]$. To date, several studies have proved the value of clinical CSF mNGS for finding out pathogens, improving diagnosis of Meningitis and Encephalitis [8, 14, 15]. However, it is 
unclear that the diagnostic performance of mNGS in pediatric patients with suspected CNS infection and empirical treatment. Therefore, we conducted a pediatric retrospective study to explore the clinical impact and performance of mNGS in CNS infection patients.

\section{Methods}

\section{Patients and data}

This study was a single-center, retrospective review of all patients with CSF mNGS testing at Children's Hospital of Hebei Province from April 5, 2019 to December 5, 2020. The Children's Hospital is a large tertiary teaching hospital. Most of the patients were transferred from other hospitals. Patients were enrolled based on a particular exposure (i.e., suspected CNS infectious patients without pathogenic evidence and received empirical treatment (Fig. 1)). Patients who were older than 18 years old were excluded. The patients' CSF was collected when a lumbar puncture was performed after admission. All of the patients' details were obtained from medical records. Clinical details were obtained, including demographic information, symptoms, clinical diagnosis, C-reactive protein (CRP), complete blood count, examinations of cerebrospinal fluid (routine biochemical, culture, smear, PCR, antibody, mNGS), and treatment. Details about CSF mNGS test (BGI China) including results, interval (in days) from illness onset to CSF collection, lengths of empirical treatment (duration of antimicrobial use before the mNGS test), turnaround time (time from collection to return result), and clinical impacts (including new antimicrobial, antimicrobial de-escalation, no change) were evaluated. Clinically relevant organism identified from CSF mNGS was assessed relative to final overall diagnosis (CNS infection versus nonCNS infection). All information was collected by two independent researchers. Any discrepancies between the two researchers were resolved by consensus or, if necessary, a third-party clinician adjudicated by consulting the medical record. All of patients were classified into two groups in the final diagnosis: CNS infection group and non-CNS infection group. mNGS test was defined as positivity if it identified an organism. Otherwise, the mNGS test was defined as negative. In this way, CNS infection patients were divided into two groups (mNGS positive group and mNGS negative group). This retrospective study was approved by Children's Hospital of Hebei Province ethics committee (Approval number: 2018014). A signed, written informed consent form was attained from the patient's guardian. All methods were performed in accordance with the relevant guidelines and regulations.

\section{mNGS of CSF}

CSF specimens $(0.5 \mathrm{ml})$ were collected from patients according to standard aseptic procedures, snap frozen, stored at $-20 \circ \mathrm{C}$, and subjected to $\mathrm{mNGS}$ within $24 \mathrm{~h}$. The process of CSF mNGS consisted sample processing, nucleic acid (DNA and RNA) extraction, followed by library generation and bioinformatic pipeline analysis. After samples were received in the clinical laboratory, sample were processed by adding glass beads to CSF samples, followed by vigorous agitation. Then extracted DNA/ RNA was fragmented to yield 150-bp to 200-bp fragments, and DNA fragments were constructed through an end-repair method. Quality-controlled libraries were sequenced on a BGISEQ-500/50 platform (BGI-Tianjin, Tianjin, China). 
For each sample, an average of 20 million reads was obtained. High-quality sequencing data were generated by removing low-quality and short (length $<35 \mathrm{bp}$ ) reads, after that computational subtraction of human host sequences were mapped to a human reference genome ( $\mathrm{YH}$ sequences and hg19) using Burrows-Wheeler alignment. Nonhuman sequences were mapped to categorization reference databases downloaded from NCBI (ftp://ftp.ncbi.nlm.nih.gov/genomes/), which included the genome sequences of 3446 bacterial species ( 45 species of mycoplasma/chlamydia and 104 species of Mycobacterium tuberculosis), 206 fungal species, 1515 viral species, and 140 parasites connected to human diseases.

\section{Diagnostic assessment of mNGS}

Definitive clinical diagnoses of the participants who completed the study were adjudicated by retrospective. The diagnosis of CNS infection was done according to the diagnosis of CNS infections written by Singhi et al [16]. The diagnosis of autoimmune encephalitis was carried out in accordance with the clinical diagnosis of autoimmune encephalitis published by Graus et al [17]. The diagnosis of epilepsy was made in accordance with the clinical diagnostic criteria for epilepsy [18].

The clinical utility of mNGS was evaluated through the following steps. Firstly, clinical characteristics were compared between CNS infections patients with positive and negative mNGS groups. Second, to evaluate whether mNGS test had a clinical impact. Clinical impact was defined as the following conditions: 1) added new targeted antimicrobial, 2) de-escalation of antibiotic therapy, according mNGS test results. Finally, diagnostic performance of mNGS results was calculated on the basis of the following two approaches [19]. In the absence of a gold standard for mNGS results, positive percent agreement (PPA: agreement between mNGS test and CNS infection diagnosis) and negative percent agreement (NPA: agreement between negative mNGS test and diagnosis of non-CNS infection) were reported instead of sensitivity and specificity. In addition, the proportion of true positives out all positive mNGS findings and proportion of true negatives out of all negative mNGS tests were described. mNGS tests often identified two or more organisms, which can be divided into two categories: clinically relevant and clinically irrelevant organisms depending on whether the organism had been recognized as causative agents. One method we counted one CSF sample as one test. An organism in one CSF sample test was clinically relevant, mNGS test was defined as true positive. Another method was adopted: each organism identified was counted and assessed independently. This method provides more detail for mNGS findings by separately assessing each organism.

\section{Statistical analyses}

For categorical data, Fisher's exact test or chi-square test was used as appropriate. Continuous data were compared by Mann-Whitney U test or Student's t-test. For baseline characteristics and CSF laboratory tests, continuous variants were described by means when they conformed to the Kolmogorov-Smirnov test and by medians when not. A p value $<0.05$ was considered significantly, and all tests were 2-tailed. All statistical analyses were performed using SPSS version 26 (IBM SPSS Statistics for Windows, Armonk, NY). 


\section{Results}

\section{Patient Characteristics}

Between April 5, 2019, and December 5, 2020, a total of 60 patients were screened in this study (Fig. 1). A total of 57 patients met the enrollment criteria and completed the study. The average age of the 57 patients (61.4\% were male) was 5.3 years (median, 5; range, 0.2 to 14$)$; 23 patients (40.4\%) were 3 years of age or younger. The cohort included patients with CNS meningitis (39 patients: 23 bacterial infections, 2 viral infections, 2 fungal infections, 12 suspected CNS infections) and non-CNS meningitis (18 patients: 15 autoimmune encephalitis, 1 epilepsy, 2 cavernous intracranial hemangiomas). In the CNS infections group, age, CSF glucose level, and turnaround time of mNGS were significantly lower than those in the non-CNS infections group (table1). The proportion of mNGS positive and CRP was significantly higher in the CNS infections than in the non-CNS infections group. According to results of mNGS, CNS infection patients were divided into two groups ( $m$ NGS positive group $n=28$ and $m$ NGS negative group $n=11$ ). In mNGS positive group, length of hospital stay was significantly longer than negative group. CRP, CSF WBC and the proportion of patients with fever were higher in the mNGS positive group than those in the negative group, but there was no statistical significance. Meanwhile, lengths of empirical treatment in mNGS positive group were shorter than that in negative group but not reached statistical significance $(P=0.246$, Table 2).

Table 1 Baseline characteristic of participants

\begin{tabular}{|c|c|c|c|}
\hline & $\begin{array}{l}\text { CNS infection } \\
(\mathrm{n}=39)\end{array}$ & $\begin{array}{l}\text { Non-CNS infection } \\
(\mathrm{n}=18)\end{array}$ & $\begin{array}{c}P \\
\text { value }\end{array}$ \\
\hline CNS infection (n) & 39 & - & - \\
\hline Bacterial infection & 23 & - & - \\
\hline Viral infection & 2 & - & - \\
\hline Fungal infection & 2 & - & - \\
\hline Suspected CNS infections & 12 & - & - \\
\hline Non-CNS infection (n) & - & 18 & - \\
\hline Autoimmune encephalitis & - & 15 & - \\
\hline Epilepsy & - & 1 & - \\
\hline Cavernous intracranial hemangioma & - & 2 & - \\
\hline Male sex, n (\%) & $23(59.0)$ & $12(66.7 \square$ & \\
\hline Age, year, median (interquartile range) & $4.00(0.96,6.50)$ & $7.5(4.00,9.00)$ & $0.022 *$ \\
\hline \multicolumn{4}{|l|}{ Clinical manifestations, $\mathrm{n}(\%)$} \\
\hline Fever & $35(89.7)$ & $14(77.8)$ & 0.424 \\
\hline Seizure & $9(23.1)$ & $6(33.3)$ & 0.621 \\
\hline Headache & $17(43.6)$ & $8(44.4)$ & 0.952 \\
\hline $\begin{array}{l}\text { Time from onset to CSF mNGS collection, day, median } \\
\text { (IQR) }\end{array}$ & $15.00(6.50,22.0)$ & $9.50(8.00,25.00)$ & 0.979 \\
\hline \multicolumn{4}{|l|}{ Blood laboratory examination } \\
\hline C-reaction protein, $\mathrm{mg} / \mathrm{L}$, median (IQR) & $2.75(0.79,14.49)$ & $0.69(0.25,3.8)$ & $0.024 *$ \\
\hline WBC, $\times 10^{9} / \mathrm{L}$, median (IQR) & $10.6(6.85,12.30)$ & $7.5(6.3,11.7)$ & 0.279 \\
\hline Neutrophil, \%, mean \pm SD & $57.56 \pm 2.91$ & $63.89 \pm 3.18$ & 0.193 \\
\hline \multicolumn{4}{|l|}{ CSF laboratory examination } \\
\hline WBC, $\times 10^{6} / \mathrm{L}$, median (IQR) & $\begin{array}{c}64.00(24.50 \\
264.5)\end{array}$ & $47.00(14.00,93.00)$ & 0.135 \\
\hline Glucose, mmol/L, median (IQR) & $3.02(2.59,3.08)$ & $3.45(2.98,3.84)$ & $0.008 *$ \\
\hline Protein, g/L, median (IQR) & $0.34(0.29,0.52)$ & $0.26(0.19,0.37)$ & 0.075 \\
\hline Chlorine, mmol/L, median (IQR) & $121.7(120.4,122.4)$ & $121.6(118.7,124.7)$ & 0.816 \\
\hline mNGS positive, $\mathrm{n}(\%)$, median (IQR) & $28(71.8)$ & $7(38.9)$ & $0.018 *$ \\
\hline Turnaround time of mNGS, day, median (IQR) & $2.00(2.00,2.50)$ & $3.00(2.00,4.00)$ & $0.026 *$ \\
\hline Length of hospital stay, day, median (IQR) & $21.70(14.5,28.0)$ & $21.7(19.0,27.0)$ & 0.705 \\
\hline
\end{tabular}


IQR: interquartile range; SD: standard deviation; * $p<0.05$

Table 2 Comparison of patients with CNF infection according mNGS positive or negative

\begin{tabular}{|c|c|c|c|}
\hline & $\begin{array}{c}\text { mNGS positive } \\
\mathrm{N}=28\end{array}$ & $\begin{array}{c}\text { mNGS negative } \\
\mathrm{N}=11 \\
\end{array}$ & $P$ value \\
\hline Male sex, n (\%) & $16(63.60)$ & $7(57.10)$ & 0.993 \\
\hline Age, year, median (IQR) & $5.5(1.00,8.00)$ & $4.00(0.67,7.50)$ & 0.794 \\
\hline Clinical manifestations $\mathrm{n}(\%)$ & & & \\
\hline Fever & $26(92.90)$ & $9(81.80)$ & 0.562 \\
\hline Seizure & $7(25.00)$ & $2(18.20)$ & 1.000 \\
\hline Headache & $14(50.00)$ & $3(27.30)$ & 0.288 \\
\hline $\begin{array}{l}\text { Time from onset to CSF mNGS collection, day, median } \\
\text { (IQR) }\end{array}$ & $14(6,21.5)$ & $18(9,24)$ & 0.450 \\
\hline lengths of empirical treatment, day, median (IQR) & $6(3.00,10.00)$ & $10(5.50,18.00)$ & 0.246 \\
\hline Blood laboratory examination & & & \\
\hline C-reaction protein, mg/L, median (IQR) & $6.22(0.69,14.49)$ & $1.80(1.07,12.39)$ & 0.914 \\
\hline WBC, $\times 10^{9} / \mathrm{L}$, median (IQR) & $10.15(6.85,12.95)$ & $10.60(7.75,11.45)$ & 0.842 \\
\hline Neutrophil, \%, mean \pm SD & $58.66 \pm 2.89$ & $54.76 \pm 7.47$ & 0.555 \\
\hline $\begin{array}{l}\text { CSF laboratory examination } \\
\text { WBC, } \times 10^{6} / \mathrm{L} \text {, median (IQR) }\end{array}$ & $\begin{array}{l}127.00(35.00 \square \\
276.00)\end{array}$ & $53.00(19.50,135.50)$ & 0.331 \\
\hline Glucose, mmol/L, median (IQR) & $3.03(2.59,3.17)$ & $2.90(2.56,3.04)$ & 0.528 \\
\hline Protein, $\mathrm{g} / \mathrm{L}$, median (IQR) & $0.35(0.29,0.50)$ & $0.32(0.25,0.59)$ & 0.866 \\
\hline Chlorine, mmol/L, median (IQR) & $\begin{array}{c}121.70 \\
(120.60,122.40)\end{array}$ & $\begin{array}{c}121.12 \\
(118.20,122.70)\end{array}$ & 0.315 \\
\hline CSF culture positive, $\mathrm{n}(\%)$ & $2(7.10)$ & 0 & 1.000 \\
\hline Turnaround time of mNGS, day, $\mathrm{n}$ & $2.00(2.00,2.50)$ & $2.00(2.00,2.50)$ & 0.939 \\
\hline Length of hospital stay, day, median (IQR) & $21.73(16.50,34.00)$ & $12.00(10.00,19.00)$ & $0.001 *$ \\
\hline
\end{tabular}

IQR: interquartile range; SD: standard deviation; * $p<0.05$

\section{Organisms and clinical impact of each mNGS test}

Overall, in 57patients, 65 organisms were identified by mNGS. In 22 patients, mNGS test identified no organisms. In 16 patients, mNGS test identified one organism, and in 19 patients, mNGS test identified two or more organisms (Additional Fig S1). In the mNGS positive group, 54.3\% (19/35) mNGS tests identified multiple organisms, and per mNGS test identified 1.9 organisms. Categories of organisms were compared. mNGS identified bacteria $(47.7 \%, 31 / 65)$, viruses $(20.0 \%, 13 / 65)$, fungus $(4.6 \%, 3 / 65)$, and Mycobacterium tuberculosis complex (1.5\%, 1/65) (Additional Fig S2). Table 3 summarizes the clinical impact of each mNGS test. In all of the patients, $75.4 \%$ (45 cases) had changed (addition or deescalation) in antimicrobial therapy. In the mNGS positive and negative group, the proportions of clinical impact were $88.6 \%$ and $54.5 \%$ respectively.

Table 3 Clinical impact of each CSF mNGS test

\begin{tabular}{lccc}
\hline & $\begin{array}{c}\text { Total } \\
\mathrm{N}=57\end{array}$ & $\begin{array}{c}\text { mNGS positive } \\
\mathrm{n}=35\end{array}$ & $\begin{array}{c}\text { mNGS negative } \\
\mathrm{n}=22\end{array}$ \\
\hline New antimicrobial, n (\%) & $12(21.0)$ & $12(34.3)$ & - \\
Antimicrobial de-escalation, n (\%) & $31(54.4)$ & $19(54.3)$ & $12(54.5)$ \\
No Change, n (\%) & $14(24.6)$ & $4(11.4)$ & $10(45.5)$ \\
Total clinical impact cases, n (\%) & $43(75.4)$ & $31(88.6)$ & $12(54.5)$ \\
\hline
\end{tabular}

\section{Performance of mNGS}


By each CSF mNGS test as a whole, the positive and negative percent agreement were $69.2 \%$ (95\% Cl: $54.1-84.4 \%)$ and $61.1 \%$ (95\% Cl: $36.2-86.1 \%)$, respectively, and the proportion of true positives of mNGS positives was $77.1 \%$ (95\% Cl: $62.5-91.8 \%$ ), and true negatives of negative mNGS tests was $50.0 \%$ (95\% Cl: $27.3-72.7 \%$ ) (Table 4). Seven mNGS tests identified organisms in non-CNS infection patients (Additional file: Table S1). One mNGS test identified only clinically irrelevant organisms (Additional file: Table S2), and three mNGS tests showed both clinically relevant and irrelevant organisms (Additional file: Table S4). When each organism identified was analyzed independently, the positive and negative percent agreement of mNGS were $76.2 \%$ (95\% Cl: $65.4-87.0 \%)$ and $45.8 \%$ (95\% Cl: $24.3-67.3 \%)$, respectively, and the proportion of true positives out of all mNGS positives was $73.8 \%$ (Table 5 ).

Table 4 mNGS test performance characteristics calculated by each CSF mNGS test as a whole

\begin{tabular}{|c|c|c|c|c|}
\hline & $\begin{array}{l}\text { CNS infection related to } \\
\text { mNGS test }\end{array}$ & $\begin{array}{l}\text { CNS infection not related to } \\
\text { mNGS test }\end{array}$ & Non-CNS infection & \\
\hline $\begin{array}{l}\text { mNGS } \\
\text { identified } \\
\text { organism }\end{array}$ & $\begin{array}{l}\text { Box A: true positive (TP, } \\
\text { with clinically relevant } \\
\text { organism): } 27\end{array}$ & $\begin{array}{l}\text { Box B: false positive } 1 \text { (FP1, } \\
\text { only with clinically } \\
\text { irrelevant organism): } \\
1\end{array}$ & $\begin{array}{l}\text { Box C: false positive } 2 \\
\text { (FP2, only clinically } \\
\text { irrelevant organism): } 7\end{array}$ & $\begin{array}{l}\text { True positives } \\
\text { out of mNGS } \\
\text { positives: } \\
\text { TP/ (box A } \\
+ \text { B+C): } \\
77.1 \% \\
95 \% \text { CI: } 62.5- \\
91.8 \%\end{array}$ \\
\hline $\begin{array}{l}\text { mNGS } \\
\text { identified } \\
\text { no } \\
\text { organism }\end{array}$ & \multicolumn{2}{|l|}{$\begin{array}{l}\text { Box D: False negative (FN): } \\
11\end{array}$} & $\begin{array}{l}\text { Box E: True } \\
\text { negative (TN): } \\
\mathbf{1 1}\end{array}$ & $\begin{array}{l}\text { True negatives } \\
\text { out of } \\
\text { mNGS } \\
\text { Negatives: } \\
\text { TN/ (box } \\
\text { D+E): } \\
50.0 \% \\
95 \% \text { CI: } 27.3- \\
72.7 \%\end{array}$ \\
\hline & $\begin{array}{l}\text { Positive percent } \\
\text { agreement: } \\
\text { TP/ (box A+B+D): } \\
69.2 \% \\
\text { 95\%CI: } 54.1-84.4 \% \\
\end{array}$ & & $\begin{array}{l}\text { Negative percent } \\
\text { agreement: } \\
\text { TN/ (box C+E): } \\
61.1 \% \\
\text { 95\%CI: } 36.2-86.1 \% \\
\end{array}$ & \\
\hline
\end{tabular}

Table 5 mNGS test performance characteristics calculated by each organism (total 65 organisms, 22 tests with no organism) 


\begin{tabular}{|c|c|c|c|c|}
\hline & $\begin{array}{l}\text { CNS infection } \\
\text { related to mNGS } \\
\text { test }\end{array}$ & $\begin{array}{l}\text { CNS infection not related to } \\
\text { mNGS test }\end{array}$ & Non-CNS infection & \\
\hline $\begin{array}{l}\text { mNGS } \\
\text { identified } \\
\text { organism }\end{array}$ & $\begin{array}{l}\text { Box A: true } \\
\text { positive } \\
\text { organism (TP): } \\
48\end{array}$ & $\begin{array}{l}\text { Box B: false positive 1 (FP1, } \\
\text { clinically irrelevant } \\
\text { organism): } 4\end{array}$ & $\begin{array}{l}\text { Box C: false positive } 2 \text { (FP2, } \\
\text { clinically irrelevant } \\
\text { organism): } 13\end{array}$ & $\begin{array}{l}\text { True positives } \\
\text { out of mNGS } \\
\text { positives: } \\
\text { TP/ (box A } \\
+ \text { B+C): } \\
73.8 \% \\
95 \% \text { CI: } 62.9- \\
84.8 \%\end{array}$ \\
\hline \multirow[t]{2}{*}{$\begin{array}{l}\text { mNGS } \\
\text { identified no } \\
\text { organism }\end{array}$} & \multicolumn{2}{|c|}{$\begin{array}{l}\text { Box D: False negative (FN): } \\
11\end{array}$} & $\begin{array}{l}\text { Box E: True } \\
\text { negative (TN): } \\
\mathbf{1 1}\end{array}$ & $\begin{array}{l}\text { True negatives } \\
\text { out of } \\
\text { mNGS } \\
\text { Negatives: } \\
\text { TN/ (box D+E): } \\
50.0 \% \\
95 \% \text { CI: } 27.3- \\
72.7 \%\end{array}$ \\
\hline & $\begin{array}{l}\text { Positive percent } \\
\text { agreement: } \\
\text { TP/ (box } \\
\text { A+B+D): } \\
76.2 \% \\
95 \% \text { CI: } \\
87.0 \% \\
\end{array}$ & & $\begin{array}{l}\text { Negative percent } \\
\text { agreement: } \\
\text { TN/ (box C+E): } \\
45.8 \% \\
\text { 95\%CI: } 24.3-67.3 \%\end{array}$ & \\
\hline
\end{tabular}

\section{Discussions}

In this study, we evaluated the test performance characteristics of mNGS for diagnosing neurologic infections in a series of pediatric patients with empirical treatment before the time of enrollment. And we also assessed the clinical usefulness of mNGS. Thus, we described the real-life performance of CSF mNGS test in a difficult-to-diagnose pediatric patient for whom the essay is most likely to be performed. We assessed the clinical relevance of each mNGS test and each organism detected by mNGS, which substantially impacted the proportion of PPA for diagnosing neurologic infections (69.2\% for per-test assessment versus $76.2 \%$ for per-organism assessment). In this study, the mNGS assay identified most potential pathogens in CNF infection patients. A total of 26 infections were diagnosed solely by metagenomic NGS. It is notable that 43 of these 57 cases had a clinical effect. These findings demonstrated the potential usefulness of CSF mNGS test in these pediatric patients.

This study indicated that mNGS held diagnostic advantages over CSF culture for pediatric patients who had received empirical antimicrobial treatment. As there is currently no gold standard for mNGS results, PPA and NPA were reported instead of sensitivity and specificity. The clinical laboratory validated that PPA and NPA of CSF mNGS were $>95 \%,>80 \%$ respectively, compared to the clinical microbiologic results [20]. Our results are consistent with previous literature reports, which showed that mNGS identified multiple pathogens successfully, and demonstrated that mNGS was most useful in patients with a CSF abnormality [15]. Additionally, an adult study found that mNGS provided a higher detection rate 
compared to culture in patients with empirical therapy in suspected CNS infection, and they indicated that empirical antibiotics use did not affect the detection rate of mNGS [21]. However, effective treatment could reduce the detection rate of CSF culture in meningitis (11\%) [22]. The possible reasons for the above phenomenon are explained as follows. Culture needs the existence of livable pathogens and therefore is easily affected by the administration of antimicrobial treatment. On the other hand, mNGS requires only DNA fragments of microorganisms, which might be unaffected by treatment. Nevertheless, low concentrations of microorganisms may lead to false-negative results of mNGS [8]. This may also account for this difference observed in our studies, although we had no information on the organism concentrations. Our data found that CNS infection patients in the mNGS negative group had used antibiotics for a long time and had low inflammation markers like CRP and CSF WBC.

The diagnosis of this study exposed the challenge of data interpretation. In our study, three cases found clinically relevant and irrelevant organism, one case only found clinically irrelevant organism, and seven cases found false positive of mNGS in non-CNF infection (Additional file: Table S1). Autoimmune encephalitis was mainly established by clinical presentation, antibodies (CSF HSV PCR, VZV PCR, EBV PCR, anti-NMDAR antibody, anti-MOG antibody), and the response of treatment. Post-herpetic or EBV or other organisms. Autoimmune encephalitis could arise. A recent adult case report found that the antiNMDAR antibody (IgG) was identified positively on day 11 after herpes simplex virus infection [23]. This possibility must be recognized by clinicians, especially those with symptoms that occurred after remission [24-26].

Our results suggest that mNGS should be an effective tool to guide clinical decisions for antibiotic treatment. When mNGS identified additional organisms or none organism, changes in therapy occurred in the majority $(75.4 \%)$ of cases. Wilson et al. reported that in 13 patients identified by mNGS, 7 cases' medical treatment was adjusted according to mNGS results [8]. The results of $\mathrm{mNGS}$ can also be valuable even when no organism was detected, about half of the patients with mNGS negative $(12 / 22)$ had antimicrobial de-escalation, considering ruling out co-infections.

There were some limitations in our study.

First, our study had a relatively small sample size.

This may be the cause of that many results observed a certain trend did not achieve statistical significance. Consequently, a larger sample size research needs to be conducted in further. Second, in these patients, medication dosage and time were not uniform. The inconsistent empirical treatment may come from different hospitals in the region. Thus, it appears that mNGS is advantageous in a real-world healthcare setting. Third, this is a single central retrospective study, in which selective bias in data collection, limit generalizability, and lack of antibiotic usage detail may restrict the wide application of our results in other hospitals.

\section{Conclusion}


In summary, our major findings included performance characteristics of CSF mNGS test with $76.2 \%$ of the organisms identified as clinically relevant and clinical effects with $75.4 \%$ cases $(45 / 57)$ who had changed (addition or de-escalation) in antimicrobial therapy. Therefore, we suggest that mNGS should be used more in suspected CNS infection patients with antibiotic treatment in the future. Nonetheless, there is still a challenge to interpret data of mNGS for pediatric doctors in guiding clinical treatment. Despite the insights in our study regarding CSF mNGS test performance and utility, further research will be vital to recognize how to optimally integrate mNGS into the CNS infectious diseases diagnostic work up.

\section{List Of Abbreviations}

CNS: central nervous system; CSF: Cerebrospinal fluid; mNGS: Metagenomic next generation sequencing; PPA: positive percent agreement; NPA: negative percent agreement; EBV, Epstein-Barr virus; HSV, herpes simplex virus; VZV, varicella zoster virus; WBC, white blood cell; CRP: C-reactive protein.

\section{Declarations}

\section{Ethics approval and consent to participate}

This study was approved by the Ethics Committee of Children's Hospital of Hebei Province (Approval number: 2018014). A signed, written informed consent form was attained from the patient's guardian

\section{Consent for publication}

Not applicable.

\section{Availability of data and materials}

The data sets generated and/or analyzed during the current study are not publicly available due to the data privacy requirements of the ethics committees, but are available from the corresponding author on reasonable request and approval from the ethics committees in all institutions.

\section{Competing interests}

The authors declare that they have no competing interests.

\section{Funding}

Supported by Science and Technology Fund, Governmental Department of Health, Hebei Province, China, No. PMID:(182777153)

\section{Authors' contributions}

Su-Zhen Sun designed the study. Meng-Xiao Ding, Jing-Ru Xue and Dan-Ni Yan contributed to acquisition of clinical data. Bin-Fang Guo conducted analyses and wrote the manuscript. All of the authors read and 
approved the final article.

\section{Acknowledgments}

We thank all the patients who donate their samples.

\section{References}

1. Jmor F, Emsley HC, Fischer M, Solomon... T: The incidence of acute encephalitis syndrome in Western industrialised and tropical countries. Virology Journal 2008, 5:134. doi:10.1186/1743-422X5-134

2. Granerod J, Ambrose HE, Davies NW, Clewley JP, Walsh AL, Morgan D, Cunningham R, Zuckerman M, Mutton KJ, Solomon T et al: Causes of encephalitis and differences in their clinical presentations in England: a multicentre, population-based prospective study. Lancet Infect Dis 2010, 10(12):835-844. doi:10.1016/s1473-3099(10)70222-x

3. Granerod J, Ambrose HE, Davies NW, Clewley JP, Walsh AL, Morgan D, Cunningham R, Zuckerman M, Mutton KJ, Solomon T et al: Causes of encephalitis and differences in their clinical presentations in England: a multicentre, population-based prospective study. Lancet Infectious Diseases 2010, 10(12):835-844. doi:10.1016/S1473-3099(10)70222-X

4. Glaser CA, Honarmand S, Anderson LJ, Schnurr DP, Forghani B, Cossen CK, Schuster FL, Christie LJ, Tureen aJH: Beyond Viruses: Clinical Profiles and Etiologies Associated with Encephalitis. Clinical Infectious Diseases An Official Publication of the Infectious Diseases Society of America 2006, 43(12):1565-1577

5. Glaser CA, Honarmand S, Anderson LJ, Schnurr DP, Forghani B, Cossen CK, Schuster FL, Christie LJ, Tureen JH: Beyond viruses: clinical profiles and etiologies associated with encephalitis. Clin Infect Dis 2006, 43(12):1565-1577. doi:10.1086/509330

6. Leber AL, Everhart K, Balada-Llasat JM, Cullison J, Daly J, Holt S, Lephart P, Salimnia H, Schreckenberger PC, DesJarlais S et al: Multicenter Evaluation of BioFire FilmArray Meningitis/Encephalitis Panel for Detection of Bacteria, Viruses, and Yeast in Cerebrospinal Fluid Specimens. J Clin Microbio/ 2016, 54(9):2251-2261. doi:10.1128/jcm.00730-16

7. Donovan J, Thu DDA, Phu NH, Dung VTM, Quang TP, Nghia HDT, Oanh PKN, Nhu TB, Chau NVV, Ha VTN et al: Xpert MTB/RIF Ultra versus Xpert MTB/RIF for the diagnosis of tuberculous meningitis: a prospective, randomised, diagnostic accuracy study. Lancet Infect Dis 2020, 20(3):299-307. doi:10.1016/s1473-3099(19)30649-8

8. Wilson MR, Sample HA, Zorn KC, Arevalo S, Yu G, Neuhaus J, Federman S, Stryke D, Briggs B, Langelier $\mathrm{C}$ et al: Clinical Metagenomic Sequencing for Diagnosis of Meningitis and Encephalitis. $N$ Engl J Med 2019, 380(24):2327-2340. doi:10.1056/NEJMoa1803396

9. Xing XW, Zhang JT, Ma YB, Zheng N, Yang F, Yu SY: Apparent performance of metagenomic nextgeneration sequencing in the diagnosis of cryptococcal meningitis: a descriptive study. J Med 
Microbio/ 2019, 68(8):1204-1210. doi:10.1099/jmm.0.000994

10. Fang $M$, Weng $X$, Chen $L$, Chen $Y$, Chi $Y$, Chen W, Hu Z: Fulminant central nervous system varicellazoster virus infection unexpectedly diagnosed by metagenomic next-generation sequencing in an HIV-infected patient: a case report. BMC Infect Dis 2020, 20(1):159. doi:10.1186/s12879-020-4872-8

11. Han D, Li Z, Li R, Tan P, Zhang R, Li J: mNGS in clinical microbiology laboratories: on the road to maturity. Crit Rev Microbio/ 2019, 45(5-6):668-685. doi:10.1080/1040841x.2019.1681933

12. Forbes JD, Knox NC, Jennifer R, Franco P, Aleisha R: Metagenomics: The Next Culture-Independent Game Changer. Frontiers in Microbiology 2017, 8:1069. doi:10.3389/fmicb.2017.01069

13. Brittany, Goldberg, Heike, Sichtig, Chelsie, Geyer, Nathan, Ledeboer, George, M.: Making the Leap from Research Laboratory to Clinic: Challenges and Opportunities for Next-Generation Sequencing in Infectious Disease Diagnostics. Mbio 2015, 6(6):e01888-01815. doi:10.1128/mBio.01888-15

14. Duan H, Li X, Mei A, Li P, Liu Y, Li X, Li W, Wang C, Xie S: The diagnostic value of metagenomic

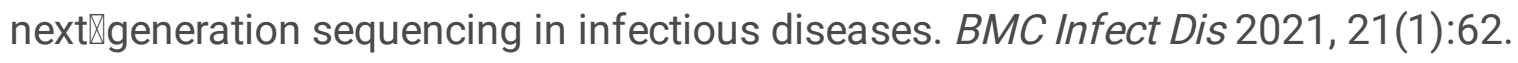
doi:10.1186/s12879-020-05746-5

15. Haston JC, Rostad CA, Jerris RC, Milla SS, McCracken C, Pratt C, Wiley M, Prieto K, Palacios G, Shane AL et al: Prospective Cohort Study of Next-Generation Sequencing as a Diagnostic Modality for Unexplained Encephalitis in Children. J Pediatric Infect Dis Soc 2020, 9(3):326-333. doi:10.1093/jpids/piz032

16. Singhi S, Angurana SK: Principles of Management of Central Nervous System Infections. Indian J Pediatr 2019, 86(1):52-59. doi:10.1007/s12098-017-2583-y

17. Graus F, Titulaer MJ, Balu R, Benseler S, Bien CG, Cellucci T, Cortese I, Dale RC, Gelfand JM, Geschwind $\mathrm{M}$ et al: A clinical approach to diagnosis of autoimmune encephalitis. Lancet Neurol 2016, 15(4): 391-404. doi:10.1016/s1474-4422(15)00401-9

18. Fisher RS, Acevedo C, Arzimanoglou A, Bogacz A, Cross JH, Elger CE, Engel J, Jr., Forsgren L, French JA, Glynn M et al: ILAE official report: a practical clinical definition of epilepsy. Epilepsia 2014, 55(4):475-482. doi:10.1111/epi.12550

19. Lee RA, Al Dhaheri F, Pollock NR, Sharma TS: Assessment of the Clinical Utility of Plasma Metagenomic Next-Generation Sequencing in a Pediatric Hospital Population. J Clin Microbio/ 2020, 58(7): e00419-00420. doi:10.1128/jcm.00419-20

20. Miller S, Naccache SN, Samayoa E, Messacar K, Arevalo S, Federman S, Stryke D, Pham E, Fung B, Bolosky WJ et al: Laboratory validation of a clinical metagenomic sequencing assay for pathogen detection in cerebrospinal fluid. Genome Res 2019, 29(5):831-842. doi:10.1101/gr.238170.118

21. Zhang Y, Cui P, Zhang HC, Wu HL, Ye MZ, Zhu YM, Ai JW, Zhang WH: Clinical application and evaluation of metagenomic next-generation sequencing in suspected adult central nervous system infection. J Transl Med 2020, 18(1):199. doi:10.1186/s12967-020-02360-6

22. Nigrovic LE, Malley R, Macias CG, Kanegaye JT, Moro-Sutherland DM, Schremmer RD, Schwab SH, Agrawal D, Mansour KM, Bennett JE et al: Effect of antibiotic pretreatment on cerebrospinal fluid 
profiles of children with bacterial meningitis. Pediatrics 2008, 122(4): 726-730.

doi:10.1542/peds.2007-3275

23. Hu S, Lan T, Bai R, Jiang S, Cai J, Ren L: HSV encephalitis triggered anti-NMDAR encephalitis: a case report. Neurol Sci 2021. doi:10.1007/s10072-020-04785-9

24. Armangue T, Spatola M, Vlagea A, Mattozzi S, Cárceles-Cordon M, Martinez-Heras E, Llufriu S, Muchart J, Erro ME, Abraira $L$ et al: Frequency, symptoms, risk factors, and outcomes of autoimmune encephalitis after herpes simplex encephalitis: a prospective observational study and retrospective analysis. Lancet Neurol 2018, 17(9): 760-772.doi:10.1016/s1474-4422(18)30244-8

25. Dorcet G, Benaiteau M, Bost C, Mengelle C, Bonneville F, Martin-Blondel G, Pariente J: Two Cases of Late-Onset Anti-NMDAr Auto-Immune Encephalitis After Herpes Simplex Virus 1 Encephalitis. Front Neuro/ 2020, 11:38. doi:10.3389/fneur.2020.00038

26. Linnoila J, Pulli B, Armangué T, Planagumà J, Narsimhan R, Schob S, Zeller MWG, Dalmau J, Chen J: Mouse model of anti-NMDA receptor post-herpes simplex encephalitis. Neurol Neuroimmunol Neuroinflamm 2019, 6(2):e529. doi:10.1212/nxi.0000000000000529

\section{Figures}




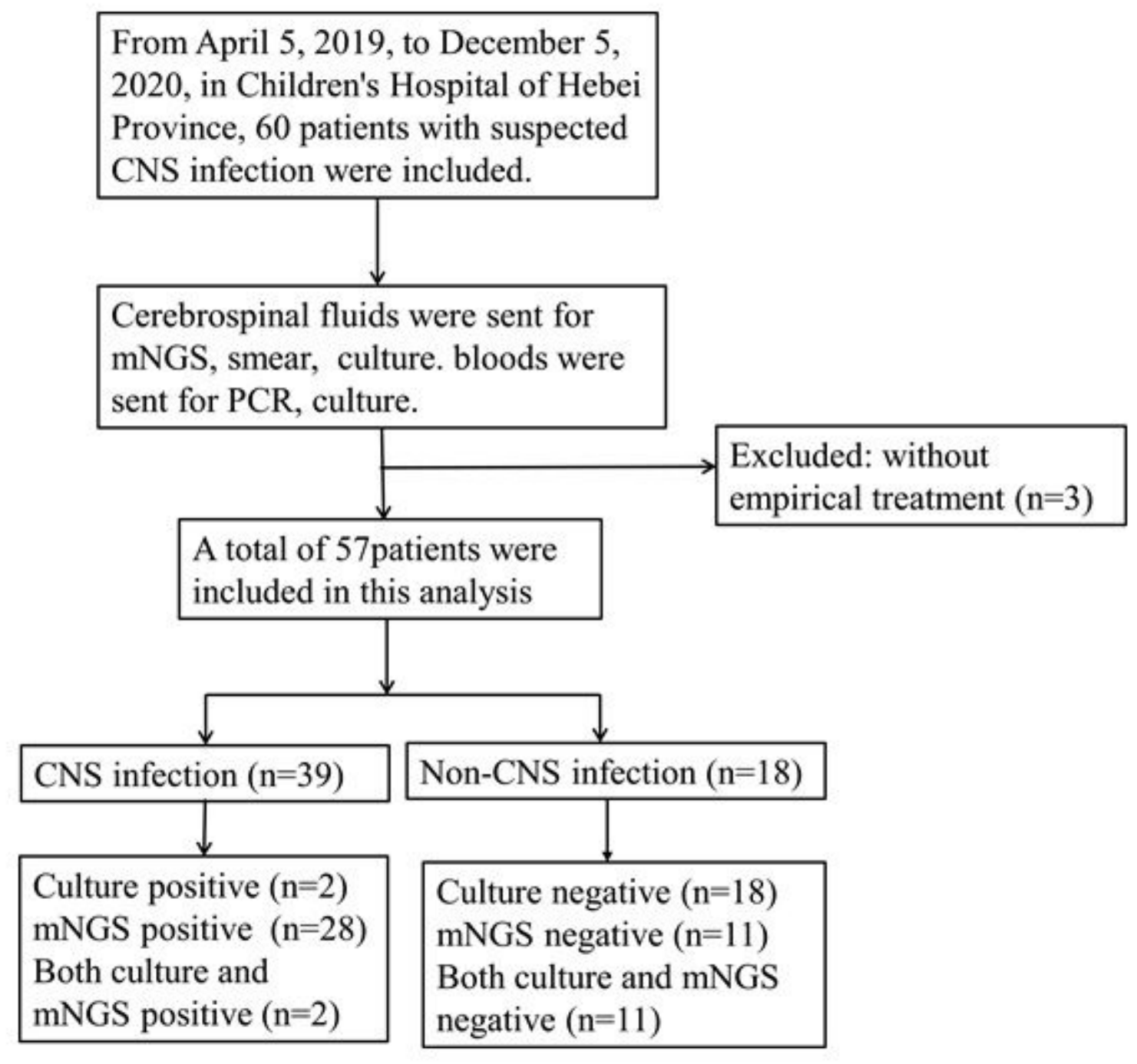

Figure 1

Flow diagram of cases inclusion and exclusion

\section{Supplementary Files}

This is a list of supplementary files associated with this preprint. Click to download.

- Additionalfile.docx 